\title{
Cardiovascular diseases and Alzheimer's disease: A potential relationship
}

\author{
Koutsouraki $\mathrm{E}^{* *}$ and Michmizos $\mathrm{D}^{2}$ \\ ${ }^{1} 1^{\text {st }}$ Department of Neurology, Medical School, Aristotle University, AHEPA Hospital, St. Kyriakidi 1, Thessaloniki, Greece \\ ${ }^{2}$ University of Thessaly, Volos, Greece
}

\begin{abstract}
The study of the relationship between cardiovascular diseases (CVDs) and Alzheimer's disease (AD) can be complicated. The diagnostic criteria of AD usually exclude patients with any clinical CVD (the combination of CVD and dementia leading more strongly towards a diagnosis of multi-infarction dementia rather than in AD). Nevertheless, there is strong evidence supporting the notion that CVDs contributes at least to the onset of AD, as demonstrated by numerous autopsy studies. The incidence of microvascular ischemic episodes has been found to strongly correlate with Dementia. Nevertheless, it is not clear whether this association is due to the fact that they share common risk factors or if the CVDs are directly or indirectly involved in the pathogenesis leading to AD. Understanding the etiology of one of these diseases can lead to understanding the onset, progression and treatment of the other.
\end{abstract}

\section{Introduction}

In Western Europe and the USA society cardiovascular diseases (CVDs) are the leading cause of death for men and women over the age of 50 and 55 years, respectively. Two million people in Europe (including fifty thousand people in Greece), and 1 million people in the USA die each year from CVDs. One in three deaths worldwide and one in two deaths in the European Union and in Greece are caused by cardiovascular diseases and the main cause of CVDs is atherosclerosis propagated by the chronic state of arterial walls inflammation.

The inflammation leads to the gradual build-up of cholesterol onto the arterial walls (both extracellular and intracellular), the accumulation of dead cell components as well as other extracellular material and dead cells components, which lead to the formation of atherosclerotic plaques. These plaques can grow to such extent so that they can cause the significant narrowing of the arteries and, thus, reduce the blood flow. Furthermore, the more extensive plaques become highly sensitive and can rupture, releasing clots that can function as embolus and cause the occlusion of arteries. If the clots occlude coronary arteries they result in an acute myocardial infarction (AMI / heart attack), whereas, if they occlude arteries in the brain they result in a stroke. The identified risk factors for atherosclerosis include high cholesterol levels (especially LDL $>100 \mathrm{mg} / \mathrm{dL}$ ), first and second smoking, hypertension, diabetes, obesity and physical inactivity [1].

\section{CVDs and Alzheimer's disease common risk factors}

The study of the relationship between CVDs and Alzheimer's disease $(\mathrm{AD})$ can be complicated. The diagnostic criteria of $\mathrm{AD}$ usually exclude patients with any clinical CVD (the combination of CVD and dementia leading more strongly towards a diagnosis of multi-infarction dementia rather than in $\mathrm{AD}$ ) [2]. Nevertheless, there is strong evidence supporting the notion that CVDs contributes at least to the onset of $\mathrm{AD}$, as demonstrated by numerous autopsy studies [3-5]. The incidence of microvascular ischemic episodes has been found to strongly correlate with Dementia, as has the number of $\mathrm{AD}$ lesions $(\mathrm{OR}=4.59$ and 4.27, respectively). In a similar study with nuns, the incidence of $\mathrm{AD}$ was 11-fold higher (95\% CI 1.8-70.3) in women who also had cerebral infarctions evident in autopsy material than in those whodid not. A number of epidemiological studies shows correlation between CVDs and cognitive loss or $\mathrm{AD}$ [6].

Nevertheless, it is not clear whether this association is due to the fact that they share common risk factors or if the CVDs are directly or indirectly involved in the pathogenesis leading to $\mathrm{AD}$.

Although the risk of developing $\mathrm{AD}$ increases with age, the disease is not part of normal aging, but, instead, it is the result of disturbances in the metabolism of brain cells. The main pathological characteristic of the disease is the formation of plaques in the brain, composed of $\beta$-amyloid peptide aggregates $(A \beta)$. The majority of patients with $A D$ show various CVDs of mixed necrotomic findings, characterized by $\beta$-amyloid plaques and microinfarctions in the brain [3]. Furthermore, the patients exhibit degeneration of small blood vessels of the brain, a finding that has yet to receive a proper explanation, resulting for them to be referred as $\mathrm{AD}$ as well as CVDs patients [4-7]. While vascular disorders are closely related to the pathology of the white matter (WM), it remains unexplained how small vessel disease affects the clinical course of AD as well as CVDs [8-10].

\section{Late-onset Alzheimer disease (LOAD)}

Investigating the contribution of CVDs to in the risk of lateonset Alzheimer disease (LOAD) has led to conflicting views. Some

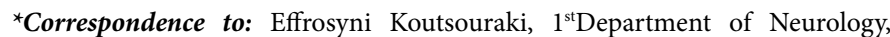
Medical School, Aristotle University, AHEPA Hospital, St. Kyriakidi 1, Thessaloniki, Greece, E-mail: efrosink@gmail.com

Key words: Alzheimer's disease, cardiovascular diseases, apolipoprotein-e, hypertension, diabetes, cholesterol

Received: October 07, 2019; Accepted: October 23, 2019; Published: November 01,2019 
researchers argue that any previous history of cardiovascular risk factors also increase the likelihood LOAD, while others take the position that such studies have unreliable results. In order to explore the relationship between the cardiovascular risk factors (diabetes, hypertension, heart disease) and a history of stroke with the onset of LOAD, information was gathered and analyzed from families with several incidents of LOAD. For the familial and sporadic LOAD, a history of stroke was significantly correlated with a high risk of the disease and attenuated the relationship between specific cardiovascular risk factors and LOAD, suggesting that genetic predisposition for LOAD is independent of the cardiovascular risk factors [11].

The progression of atherosclerosis and $\mathrm{AD}$ follow independent paths, yet with common characteristics (they manifest common epidemiological and pathophysiological data whereas both diseases have been known to respond to the same treatment). In recent years, genetic studies have shown that there are common genes involved in both diseases (the gene encoding apolipoprotein-E, genes encoding proteins involved in cholesterol metabolism, hypertension, lipid oxidation and inflammatory mechanisms). Common risk factors include hypercholesterolemia, hypertension and diabetes [12].

\section{The role of blood pressure}

The relationship between hypertension and cognitive function and dementia remains untangled, presenting a negative correlation at different stages of the disease throughout the patient's life. Some studies indicate that hypertension in middle age increases the risk of cognitive impairment whereas low blood pressure at an older age increases the chance of dementia - although this seems to be the result rather than a cause of the disease. The use of antihypertensive drugs does not appear to reduce the risk of dementia in most studies with elderly people, while studies with middle-aged people are very challenging to be completed as they require many years of monitoring until the clinical onset of the disease [13].

\section{Blood cholesterol levels and AD}

Epidemiological studies have shown that high blood cholesterol levels may contribute to the pathogenesis of $\mathrm{AD}$. One possible mechanism that has been proposed is that high blood cholesterol levels increase the risk of CVDs; whereas different one supports a direct effect between the formation and catabolism of $A \beta$ in the brain cells, particularly of $A \beta 1-42$ which is the most toxic form of $A \beta$. Patients with $\mathrm{AD}$ have indeed been found to have high levels of LDL-cholesterol and low levels of HDL-cholesterol and animals that were fed a high cholesterol diet were found to have elevated levels of $A \beta$. Moreover, the distribution and the levels of cholesterol in brain cells affect the formation and catabolism of $A \beta$. So, it should be of no surprise that some clinical studies have shown that statins, which are administered to reduce blood cholesterol levels, also protect against the $\mathrm{AD}$ and animals that received statins had lower levels of $A \beta$ [14].

In recent years, studies have been showing the homeostasis of lipids in the brain, both of cholesterol as well as sphingolipids (such as gangliosides) to be of greater importance. The lipid composition of the cell membranes, the extensive binding of $A \beta$ with the lipids, the formation of lipid rafts associated with the formation and toxicity of $\mathrm{A} \beta$, renewed the impetus to the study of the multifaceted role of lipids in amyloidogenesis, in neuroinflammation, in neuroplasticity, in mitochondrial and synaptic function as well as other key mechanisms for the occurrence of AD. Nevertheless, it remains unanswered whether disturbances of lipid homeostasis observed in $\mathrm{AD}$ is the cause or result of the disease [15-17]

\section{Diabetes and dementia}

Diabetes can increase the cognitive deficits and result in dementia, through the damage caused to the systemic blood circulation and microcirculation; however, its devastating role does not end there. There are insulin receptors in the brain, particularly in the hippocampus, the primary center of episodic memory formation. Insulin also affects the electrochemical events neurons, from the neurotransmitters associated with memory and learning, to the enzymes that participate in the metabolism of $A \beta$. Thus, the production of insulin is directly related to neuroinflammation, amyloidogenesis, oxidative stress and mitochondrial function, thereby constituting a candidate link between the endocrine and the neurodegenerative diseases [18].

\section{The role of ApoE}

Apolipoprotein-E (apoE) plays an important role in atherosclerosis and, thus, in CVDs and in AD as well, not only because of its relationship with CVDs but also participating in the pathogenesis of the disease itself. ApoEis a basic protein of the lipid transport system and it plays an important role in dyslipidemia, atherosclerosis and the pathogenesis of AD. ApoE is expressed in the liver, the brain and other tissues. The most studies of its functions are associated with lipid transport and its ability to promote the removal of cholesterol and triglycerides from the blood. Mice lacking apoE develop atherosclerotic plaques, were they fed a normal diet or one rich in lipids [19].

ApoE appears in three common isoforms (apoE2, apoE3, apoE4) in the general population. The apoE4 isoform is the major risk factor of $\mathrm{AD}$, as it has been found to reduces the age of onset of the disease and induces $A \beta$ to enter the nerve cells [20]. Since structural rearrangements in apoE affect its normal functioning, a biophysical analysis of the successive C-terminal deficient isoforms of apoE4 was carried out. The deletion of the C-terminus leads to loss of functionality, not due to the molecule becoming destabilized but due to its loss of structural plasticity [21]. The e4 allele exhibits less antioxidant activity than the e3 allele, while the e2 has the highest antioxidant activity in cell cultures. Antioxidants help protect from $\mathrm{AD}$, as the disease precipitates strong oxidizing stress; to which nerve cells are particularly sensitive [22,23].

Atherosclerosis and $\mathrm{AD}$ share common molecular mechanisms of emergence and common risk factors. Understanding the etiology of one of these diseases can help us in understand the onset, progression and treatment of the other one [24]. The risk of dementia is greater when CVDs coexists. The CogFAST study showed that the presence of three or more cardiovascular risk factors increases the risk of dementia and death by four times in the elderly survivors of stroke [25]. These observations contradict a systematic review which concluded that strokes effecting the incidence of dementia is explained by a greater part by the recurrent strokes rather than the cardiovascular risk factors [26].

\section{The contribution of inflammation}

Another pathway that leads to dementia is the suppression or impairment of inflammatory mechanisms. Besides the aging of the immune system, cerebral atrophy is associated with reduced production of anti-inflammatory cytokines by the reactive microglia and astrocytes, in response to the production IL-6 and IL-8, which was observed in patients who demonstrated cognitive disorders after a stroke [27].

\section{Conclusion}

Interestingly, the cerebral atrophy in $\mathrm{AD}, \mathrm{VAD}$, mixed dementia and dementia after stroke refer to specific areas and cells, as well as 
specific functions compared to normal controls of the same age and patients with no cognitive disorders after stroke. Brain atrophy is located in the pyramidal cells $(30-40 \%)$ of the III and V layer of the upper dorsal frontal cortex when compared to the anterior gyrus of the cingulate and orbitofrontal cortex of the frontal lobe. These findings are indicative of neuronal atrophy being independent of the presence of amyloid, neurofibrillary degeneration and tau-protein. Nevertheless, the immunoreactivity of the neurofibrillary SMI31 protein was found to be elevated in patients with dementia and was associated with reduced neuronal tumors. Theoretically, secondary neurodegeneration in the gray matter of the cortex can occur after the loss of myelin and axonal damage in the white matter $[28,29]$.

The CVDs and AD have common molecular mechanisms of emergence and common risk factors. Understanding the etiology of one of these diseases can lead to understanding the onset, progression and treatment of the other.

\section{References}

1. Amarenco P, Bogousslavsky J, Caplan LR, Donnan GA, Hennerici MG (2009) Classification of stroke subtypes. Cerebrovasc Dis 27: 493-501. [Crossref]

2. Roman GC, Tatemichi TK, Erkinjuntti T, et al. Vascular dementia: diagnostic criteria for research studies. Report of the NINDS-AIREN International Workshop. Neurology 43: $250-260$.

3. Krishnamurthi RV, Feigin VL, Forouzanfar MH, et al. (2013) I.R.F.S. Global Burden of Diseases, G.B.D.S.E. Group Global and regional burden of first-ever ischaemic and haemorrhagic stroke during 1990-2010: findings from the Global Burden of Disease Study 2010. Lancet Global Health 1: e259-e281.

4. Lee S, Shafe AC, Cowie MR (2011) UK stroke incidence, mortality and cardiovascular risk management 1999-2008: time-trend analysis from the general practice research database. BMJ Open 1: e000269.

5. Connor MD, Walker R, Modi G, Warlow CP (2007) Burden of stroke in black populations in sub-Saharan Africa. Lancet Neurol 6: 269-278.

6. Rothwell PM, Coull AJ, Giles MF, et al. (2004) Change in stroke incidence, mortality, case-fatality, severity, and risk factors in Oxfordshire, UK from 1981 to 2004 (Oxford Vascular Study). Lancet 363: 1925-1933.

7. Seshadri S, Wolf PA (2007) Lifetime risk of stroke and dementia: current concepts, and estimates from the Framingham Study. Lancet Neurol 6: 1106-1114.

8. Rothwell PM, Coull AJ, Silver LE, Fairhead JF, et al. (2005) Oxford Vascular S. Population-based study of event-rate, incidence, case fatality, and mortality for all acute vascular events in all arterial territories (Oxford Vascular Study). Lancet 366: 1773-1783.

9. O'Donnell MJ, Xavier D, Liu L, et al. (2010) Risk factors for ischaemic and intracerebral haemorrhagic stroke in 22 countries (the INTERSTROKE study): a case-control study. Lancet 376: 112-123.

10. Sacco RL, Kasner SE, Broderick JP, et al. (2013) American Heart Association Stroke Council, Anesthesia, R. Council on Cardiovascular, Intervention, C. Council on, N. Stroke, E. Council on, Prevention, D. Council on Peripheral Vascular, P.A. Council on Nutrition, Metabolism An updated definition of stroke for the 21st century: a statement for healthcare professionals from the American Heart Association/American Stroke Association. Stroke 44: 2064-2089.
11. Tosto G, Bird TD, Bennett DA, et al. (2016) National Institute on Aging Late-Onset Alzheimer Disease/National Cell Repository for Alzheimer Disease (NIA-LOAD/ NCRAD) Family Study Group.). The Role of Cardiovascular Risk Factors and Stroke in Familial Alzheimer Disease. JAMA Neurol 73: 1231-1237.

12. Gorelick PB, Scuteri A, Black SE, et al. (2011) C.o.E. American Heart Association Stroke Council, C.o.C.N.C.o.C.R. Prevention, Intervention, S. Council on Cardiovascular, Anesthesia Vascular contributions to cognitive impairment and dementia: a statement for healthcare professionals from the american heart association/ american stroke association. Stroke 42: 2672-2713.

13. Rowan E, Morris CM, Stephens S, Ballard C, Dickinson H, et al. (2005) Impact of hypertension and apolipoprotein $\mathrm{E} 4$ on poststroke cognition in subjects $>75$ years of age. Stroke 36: 1864-1868. [Crossref]

14. E Gaamouch F, Jing P, Xia J, Cai D, et al. (2016) Alzheimer's Disease Risk Genes and Lipid Regulators. J Alzheimers Dis 53: 15-29. [Crossref]

15. Koutsouraki E, Hatzifilippou E, Michmizos D, Banaki T, Costa V, et al. (2014) The probable auto-antigenic role of lipids (anti-ganglioside antibodies) in the pathogenesis of Alzheimer's disease. J Alzheimers Dis 42 Suppl 3: S163-166. [Crossref]

16. Hatzifilippou E, Koutsouraki E, Costa VG, Baloyannis SJ (2014) Antibodies against gangliosides in patients with dementia. Am J Alzheimers Dis Other Demen 29: 660666. [Crossref]

17. Hatzifilippou E, Koutsouraki E, Banaki T, et al. (2010) Antibodies against GM1 in demented patients., Am J Alzheimers Dis Other Demen 23: 274-279.

18. Ribe E, Lovestone S (2016) Insulin signaling in Alzheimer's disease and diabetes: from epidemiology to molecular links. J Intern Med. 2016 Oct 14.

19. Plump AS, Smith JD, Hayek T, Aalto-Setälä K, Walsh A, et al. (1992) Severe hypercholesterolemia and atherosclerosis in apolipoprotein E-deficient mice created by homologous recombination in ES cells. Cell 71: 343-353. [Crossref]

20. Myers RH, Schaefer EJ, Wilson PW, et al. Apolipoprotein E epsilon4 association with dementia in a population-based study: The Framingham study. Neurology 46: 673-677.

21. Chroni A, Pyrpassopoulos S, Thanassoulas A, et al. (2008) Biophysical analysis of progressive C-terminal truncations of human apolipoprotein E4: insights into secondary structure and unfolding properties. Biochemistry 47: 9071-9080.

22. Ballard CG, Morris CM, Rao H, et al. (2004) APOE epsilon4 and cognitive decline in older stroke patients with early cognitive impairment. Neurology 63: 1399-1402.

23. Hatzifilippou E, Banaki T, Traka M, Koutsouraki E, Costa V, et al. (2008) Apolipoprotein E phenotype in demented patients in Greek population. Int J Neurosci 118: 163-172. [Crossref]

24. Stampfer MJ (2006) Cardiovascular disease and Alzheimer's disease: common links. $J$ Intern Med 260: 211-223. [Crossref]

25. Allan LM, Rowan EN, Firbank MJ, et al. (2012) Long term incidence of dementia, predictors of mortality and pathological diagnosis in older stroke survivors. Brain J Neurol 134: 3716-3727.

26. Savva GM, Stephan BC (2010) Alzheimer's Society Vascular Dementia Systematic Review Group. Epidemiological studies of the effect of stroke on incident dementia: a systematic review. Stroke 41: e41-e46.

27. Chen A, Oakley AE, Monteiro M, et al. (2014) Multiplex analyte assays to characterise different dementias: brain inflammatory cytokines in post-stroke and other dementias. Neurobiol Aging 38: 56-67.

28. Foster V, Oakley AE, Slade JY, et al. (2014) Pyramidal neurons of the prefrontal cortex in post-stroke, vascular and other ageing-related dementias. Brain J Neurol 137: 2509-2521.

29. Duering M, Righart R, Wollenweber FA, et al. (2015) Acute infarcts cause focal thinning in remote cortex via degeneration of connecting fiber tracts. Neurology 84 : $1685-1692$

Copyright: (C2019 Koutsouraki E. This is an open-access article distributed under the terms of the Creative Commons Attribution License, which permits unrestricted use, distribution, and reproduction in any medium, provided the original author and source are credited. 